\section{Declarações de nascidos mortos no município de São Paulo: avaliação descritiva do preenchimento}

\section{Stillbirths registers in the municipality of São Paulo: a descritive approach to filling- out of registration forms}

Evani Marzagão Beringhs 1

Paulo Rogério Gallo 2

Alberto Olavo Advincula Reis 3

1-3 Departamento de Saúde Materno Infantil. Faculdade de Saúde Pública. Universidade de São Paulo. Av. Dr. Arnaldo, 715. São Paulo, SP, Brasil. CEP: 01246-904. E-mail: evani@beringhs.com.br.

\begin{abstract}
Objectives: to study the information contained in Stillbirth Registers (SBRs) in the Municipality of São Paulo.

Methods: the adequacy of the filling out of SBR forms was assessed on the basis of the SBRs (6722) made available by the FSEADE (Foundation for Statistical Data Analysis System), using a Data Completion Index (DCI), making it possible to compare the three years studied (2001-3). Variables relating to the mother and the fetus were included where the DCI was greater than 10\%. Education, parity, place of residence, birth type, for the mother and weight, gestational age and underlying cause of death, for the fetus.

Results: the absolute stillbirth component changed little in the first two of the three years, falling slightly in the third. The variable most frequently registered was sex (98\%), followed by place of residence (82.9\%) and parity (70\%). The data least often registered were those relating to the mother's age and schooling, $20.0 \%$ and $16.7 \%$, respectively. The underlying cause was recorded in $46.7 \%$, fetal weight in $37 \%$ and type of birth in $25.3 \%$.

Conclusions: the data demonstrate that the diffi-
\end{abstract} culty encountered in incorporating this health indicator into the traditional set of indicators is in part due to the inadequacy of the data provided on the SBR form.

Key words Fetal death, Declaration of stillbirth, Fetal mortality

\section{Resumo}

Objetivos: estudar as informações contidas nas Declarações de Nascidos Mortos (DNM) no municipio de São Paulo, Brasil.

Métodos: a partir das Declarações de Nascidos Mortos, disponibilizadas pela Fundação Sistema Estadual de Análise de Dados Estatísticos (FSEADE), estudou-se a proporção de preenchimento dos registros utilizando um Índice de Preenchimento de Dados (IPD), o que possibilitou a comparação entre os anos: 2001, 2002, 2003 (n=6722). Incluiram-se as variáveis com IPD maior que $10 \%$, relativas à mãe (idade, escolaridade, paridade, local de residência, tipo de parto) e às referentes ao feto (peso, idade gestacional e causa básica).

Resultados: o componente absoluto da natimortalidade pouco se alterou nos dois primeiros periodos e caiu levemente no último. A variável sexo foi preenchida em 98\% (maior IPD), seguida pela informação do local de residência: $82,9 \%$ e número de gestações: $70 \%$. As informações menos disponiveis foram às relativas à idade e escolaridade materna, 20,0 e 16,7\% respectivamente. A causa básica do óbito foi registrada em $46,7 \%$, enquanto o peso fetal em $37 \%$ e o tipo de parto em $25,3 \%$.

Conclusões: os dados mostram que a dificuldade em se incorporar este indicador de saúde ao conjunto dos tradicionais deve-se, em parte, à heterogeneidade no preenchimento dos campos da DNM.

Palavras-chave Natimortalidade, Declaração de Nascido Morto, Mortalidade fetal 


\section{Introdução}

A mortalidade de crianças, independentemente da idade, é utilizada como indicador da qualidade de vida das populações. ${ }^{1} \mathrm{~A}$ análise dos óbitos, durante o primeiro ano de vida, pelo coeficiente de mortalidade infantil (CMI), assumiu, nas últimas décadas, grande importância como indicador de políticas públicas de saúde. ${ }^{2}$ Embora com contrastes acentuados nas diferentes regiões e entre países, esse indicador vem declinando na sua maioria. ${ }^{3}$ À medida que os valores decrescem e estabilizam-se ao redor de um dígito por 1000 nascidos vivos, o CMI tende a perder sua capacidade de discriminar diferenças entre as condições de saúde e vida das populações, o que impõe novos desafios aos gestores das políticas públicas. Na busca por informações mais sensíveis e específicas, agregam-se ao CMI outros dados epidemiológicos e sócios-demográficos 4 e refinamse os indicadores existentes em termos de qualidade das estimativas. 5,6

Entre os indicadores das condições de vida das populações, estão aqueles relacionados às condições de saúde da mulher, principalmente os que evidenciam sua condição de saúde no ciclo gravídico-puerperal. Assim, temos observado a importância crescente da mensuração da mortalidade perinatal para avaliação das condições de assistência à saúde da mulher, 7,8 em parte como indicador da qualidade da assistência médica e em parte por representar um indicador sentinela das condições sociais que envolvem a mãe e seu concepto, 8 e que além de tudo, repercute em um ônus econômico relevante para a sociedade.

Apesar de se verificar uma tendência mundial na redução dos coeficientes de mortalidade infantil, o mesmo não ocorre com a mortalidade proporcional no período perinatal. Em países desenvolvidos, observa-se uma redução da mortalidade no período neonatal precoce, mas não nas mortes fetais, que prevalecem como a maior causa de morte perinatal.7,9 No Brasil há uma tendência de declínio das taxas de mortalidade infantil, mas verifica-se uma elevação proporcional dos óbitos perinatais. 3,10

Segundo De Lorenzi et al.10a Organização Mundial da Saúde (OMS) recomenda a partir da $10^{\mathrm{a}}$ revisão (CID-10) o registro de todo óbito de fetos com mais de 22 semanas de gestação ou peso igual ou superior a 500 g. ${ }^{11}$ Tal recomendação baixou o limite anterior de peso de $1000 \mathrm{~g}$ e a idade gestacional de 28 semanas, como pontos de corte na obrigatoriedade da notificação dos natimortos. O estabelecimento destes novos padrões epidemiológicos elevou as taxas de mortalidade perinatal10 e valorizou as taxas de natimortalidade como indicador na avaliação das condições de saúde materna das populações. ${ }^{12}$ Entretanto, os novos padrões, por não serem obrigatórios, ainda não são seguidos em muitos países, dificultando a análise comparativa destes indicadores, 3,7 principalmente nos países da América Latina, em particular no Brasil. Alguns autores referem uma boa cobertura no número de registros e uma melhor qualidade no preenchimento das notificações no Estado e Município de São Paulo. 13,14 Entretanto, é questionável a qualidade das informações em relação ao registro dos natimortos. 10,15

A morte fetal pode ocorrer durante o período gestacional ou mesmo durante o trabalho de parto sob várias circunstâncias e este fato é considerado como indicador da qualidade da assistência à saúde da mulher. Desta forma, a análise das características que a circunscrevem pode fornecer importantes direcionamentos para as políticas de saúde. 15

Propõe-se nesta abordagem descritiva avaliar o preenchimento dos dados gerais das Declarações de Nascidos Mortos, no município de São Paulo.

\section{Métodos}

Estudou-se as Declarações de Óbito Fetais (DO) do município de São Paulo, no triênio 2001-2003 $(n=6722)$. O período foi definido em função da disponibilidade de dados fornecidos pela Fundação Sistema Estadual de Análise Estatística (FSEADE).

O banco de dados foi reestruturado, sendo excluídas as perdas fetais registradas com peso menor do que $500 \mathrm{~g}$, conforme as orientações da OMS. 16

Obteve-se, também na FSEADE, o número de nascidos vivos, de óbitos de menores de um ano e os coeficientes de mortalidade infantil, para o mesmo período estudado, comparando-os entre si.

A Declaração de Óbito discrimina nove blocos de informações, a saber: Bloco I - Cartório; Bloco II - Identificação; Bloco III - Residência; Bloco IV Ocorrência; Bloco V - Óbito fetal ou menor de um ano; Bloco VI - Condições e causas do óbito; Bloco VII - Médico; Bloco VIII - Causas externas e Bloco IX - Localidade sem Médico. Neste trabalho foi verificado o preenchimento dos campos referentes ao óbito fetal, presentes no Bloco V, e o preenchimento da Causa Básica (Bloco VI).

As variáveis analisadas das declarações foram às relativas à mãe: idade, escolaridade, paridade, local de residência, tipo de parto; e às referentes ao feto: peso fetal, idade gestacional e causa básica. 
Utilizou-se de uma medida de proporção para verificar o preenchimento de dados que foi denominada Índice de Preenchimento dos Dados (IPD), ponderada em relação ao total de DO fetais. O IPD possibilita verificar e comparar a eficácia das equipes de saúde no tocante ao registro dos campos.

Por se tratar do universo das DO fetais, a análise dos valores, apresentados nas tabelas, foi feita por comparação direta dos coeficientes e taxas. Considerou-se neste estudo como Razão de natimortalidade o número de óbitos fetais com peso acima de $500 \mathrm{~g}$ por 1000 nascidos vivos. 16

\section{Resultados}

Observa-se na Tabela 1 que há uma ligeira queda nos Coeficientes de Mortalidade Infantil (mortes infantis abaixo de um ano de vida por mil nascidos vivos) nos anos estudados, contudo, a Razão de Natimortalidade mantém-se nos mesmos patamares em dois dos três períodos e apresenta uma discreta queda no ano de 2003.

$\mathrm{Na}$ Tabela 2, observa-se o número total das declarações de nascido morto a cada ano e a distribuição das variáveis segundo o IPD.

Nota-se um declínio no total de ocorrências declaradas.

Com relação ao IPD, a variável declarada com maior índice de preenchimento, foi o sexo do feto, estando presente em aproximadamente $98 \%$ das Declarações de Nascidos Mortos (DNM). Segue-se o local de residência, com $82,90 \%$.

As variáveis menos declaradas foram: a idade e escolaridade da mãe, com um IPD de 19,97 e $16,69 \%$ dos casos, respectivamente.

O peso ao nascer, variável importante por ser classificatória na viabilidade fetal, está presente em pouco mais de um terço $(37,32 \%)$ das declarações. Chama a atenção as declarações do ano de 2002, no qual o IPD do peso não atingiu $30 \%$ dos casos $(29,3 \%)$.

Quanto ao número de gestações, o IPD mostra

Tabela 1

Distribuição do Coeficiente de Mortalidade Infantil e Razão de Natimortalidade. Município de São Paulo, $2001-2003$.

\begin{tabular}{lcc}
\hline Ano & Coeficiente de Mortalidade Infantil & Razão de Mortalidade (por 1000 nascidos vivos) \\
\hline 2001 & 15,35 & 3,59 \\
2002 & 15,10 & 3,64 \\
2003 & 14,23 & 3,36 \\
Média & 14,89 & 3,53 \\
\hline
\end{tabular}

Tabela 2

Distribuição dos Índices de Preenchimento de Dados (IPD), segundo variável de estudo e ano. Município de São Paulo, 2001-2003.

\begin{tabular}{|c|c|c|c|c|c|c|c|c|}
\hline \multirow[t]{2}{*}{ IPD-ano } & \multicolumn{2}{|c|}{2001} & \multicolumn{2}{|c|}{2002} & \multicolumn{2}{|c|}{2003} & \multicolumn{2}{|c|}{ Total } \\
\hline & $\mathrm{n}$ & $\%$ & $\mathrm{n}$ & $\%$ & $\mathrm{n}$ & $\%$ & $\mathrm{n}$ & $\%$ \\
\hline Total de notificações & 2322 & 100,00 & 2300 & 100,00 & 2100 & 100,00 & 6722 & 100,00 \\
\hline Sexo & 2281 & 98,23 & 2254 & 97,91 & 2060 & 98,04 & 6595 & 98,11 \\
\hline Peso & 822 & 35,40 & 686 & 29,83 & 991 & 46,74 & 2499 & 37,32 \\
\hline Idade da mãe & 485 & 20,88 & 441 & 19,17 & 428 & 20,42 & 1343 & 19,97 \\
\hline Escolaridade da mãe & 423 & 18,21 & 344 & 14,63 & 355 & 16,90 & 1122 & 16,69 \\
\hline Causa básica & 1096 & 48,04 & 1107 & 48,13 & 941 & 44,93 & 3144 & 46,77 \\
\hline Gestação & 1943 & 83,67 & 1444 & 62,76 & 1318 & 62,76 & 4705 & 69,99 \\
\hline Local de residência & 1908 & 82,17 & 1926 & 83,48 & 1739 & 82,80 & 5573 & 82,90 \\
\hline
\end{tabular}


que o ano de 2001 apresentou o maior índice do triênio $(83,67 \%)$. Contudo, nos dois anos seguintes, as declarações reduziram-se significativamente e mantiveram-se ao redor de $62 \%$. Estes valores sintetizados em uma média no triênio ficam ao redor de $70 \%$, valor considerado alto em relação às outras informações registradas e colocam a informação sobre "número de filhos tidos" como o terceiro campo mais preenchido pelas equipes de saúde responsáveis pelas DNM.

Ainda em relação às características maternas, analisou-se o tipo de parto, se espontâneo ou operatório. Nesse campo, a ausência de informações mostrou-se a condição preponderante, pois alcançou $75 \%$ das DNM (média de preenchimento no triênio de $25,3 \%$ ).

Finalmente, o IPD sobre causa básica da ocorrência não atingiu $50 \%$ dos casos (46,77\%). Índice esse que, apesar de uma discreta piora no ano de 2003, praticamente não sofreu alteração no triênio.

\section{Discussão}

Os estudos sobre mortalidade continuam sendo importantes para avaliar os níveis de saúde das coletividades, destacando-se entre eles o coeficiente de mortalidade infantil, 2 onde ainda se observa o empenho da comunidade científica voltado a elevar sua acurácia. 17,18

Contudo, no Brasil e em outros países em desenvolvimento, os estudos ressaltam o período perinatal como momento decisivo para a viabilidade das crianças e extremamente importante para avaliar a assistência à saúde materna. 3,10 Assim, novos indicadores têm sido propostos.

Desta forma, significou um grande avanço nas estatísticas vitais, a obrigatoriedade da Declaração de Nascido Morto, ressaltando sua importância em termos de políticas públicas, na área de saúde da mulher e da criança. 10,14

Em relação ao Sistema de Informação em Mortalidade (SIM), observa-se um hiato entre a regulamentação da obrigatoriedade no registro da informação e a qualidade dos dados encontrados. 19 Neste sentido, cabe destacar a decisão da OMS em alterar a faixa de peso de $1000 \mathrm{~g}$ para $500 \mathrm{~g}$, no tocante à definição de viabilidade fetal, que elevou às ocorrências da natimortalidade, decisão que justificaria, por si só, a necessidade de revisão técnica da notificação. Do ponto de vista da política de assistência à saúde da mulher, essa decisão enfatiza, ainda mais, a atenção pré-natal como estratégia fundamental para garantir a viabilidade da criança e a saúde da mulher. 9,12

Os resultados encontrados neste estudo, entretanto, mostram uma dificuldade, vinculada tanto à qualidade como à quantidade dos registros preenchidos nas DNM, com repercussões nas análises epidemiológicas no cenário da saúde pública. O foco dessa dificuldade está alocado num patamar de difícil aceitação. Não se trata apenas de informações com análise questionável, mas sim, a pouca importância no preenchimento das notificações obrigatórias. A falta de informação leva aos questionamentos das definições que, por sua vez, ficam prejudicadas. Não se pode garantir que os números de natimortos sejam fidedignos. Com relação ao peso ao nascer, a morte fetal pode estar sendo confundida com abortamento, uma vez que o peso foi informado em menos de $50 \%$ dos casos. 20

Quanto à idade materna, informação essencial no planejamento de ações específicas para a saúde da mulher, 21 foi encontrada em apenas $20 \%$ das declarações. Destaca-se aqui a importância da assistência ao pré-natal das adolescentes,22-24 que neste contexto, da omissão de informações, impede qualquer avaliação.

O mesmo pode ser dito em relação à escolaridade materna, considerada como fator de risco gestacional21,23 e de natimortalidade, que foi informada em apenas $16,7 \%$ dos casos.

Por fim, falta destacar os desdobramentos em não se haver registro das Causas Básicas das perdas fetais. Laurenti et al.18 citam que o percentual de causas mal definidas nas DOs podia ser considerada alto ao se analisar os dados de mortalidade. Contudo, estes mesmos autores citavam, em 1990,13 o Estado de São Paulo como um daqueles no qual esta situação era a menos freqüente, quando comparada a outras regiões do país. O mesmo não pode ser dito em relação às perdas fetais, pois neste estudo a causa básica foi registrada em menos de $50 \%$ dos casos.

Nesse trabalho, voltado apenas a analisar os dados do município de São Paulo, esperava-se encontrar IPDs mais altos, compatíveis com a importância epidemiológica da informação e a estrutura das políticas de saúde oferecidas nesse município, uma vez que em São Paulo os recursos diagnósticos são tradicionalmente melhores. Segundo Szwarcwald et al.,17 a distribuição dos recursos de saúde tenderia a ser pior se estudássemos outras regiões do país. Da mesma forma ocorreria em relação às estatísticas vitais. 


\section{Referências}

1. Brasil. Ministério da Saúde. Secretaria de Atenção à Saúde. Departamento de Atenção Básica. Sistemas de Informação sobre Mortalidade (SIM) e nascidos vivos (SINASC): para os profissionais do Programa Saúde da Família. Brasília, DF; 2004.

2. Monteiro CA, Nazário CL. Declínio da mortalidade infantil e equidade social: o caso da cidade de São Paulo entre 1973 e 1993. In: Monteiro CA, organizador. Velhos e novos males da saúde no Brasil: a evolução do país e de suas doenças. São Paulo: Hucitec, Núcleo de Pesquisas Epidemiológicas em Nutrição e Saúde da USP; 1995. p. 173-85.

3. Vermelho LV, Leal AJC, Kale PL. Indicadores de saúde. In Medronho RA, organizador. Epidemiologia. São Paulo: Atheneu; 2003. p. 39-43.

4. Schramm JM, Szwarcwald CL. Diferenciais nas taxas de mortalidade neonatal e nati-mortalidade hospitalares no Brasil: um estudo com base no Sistema de Informações Hospitalares do Sistema Único de Saúde (SIH/SUS). Cad Saúde Pública. 2000; 16: 1031-40.

5. Szwarcwald CL, Leal MC, Andrade CLT. Infant mortality estimation in Brazil: what do Ministry of Health data on deaths and live births say? Cad Saúde Pública. 2002; 18: 1725-36.

6. OMS (Organização Mundial da Saúde). Programa das Nações Unidas para o Desenvolvimento: relatório de desenvolvimento humano (RDH) e Índice de Desenvolvimento Humano (IDH). Disponível em: URL: http://pnud.org.br/ idh. [2006 jul 17].

7. Cabral ACV, Rezende CAL, Celani MFS, Colosimo EA. Natimortalidade: estudo epidemiológico e etiológico. J Bras Ginecol. 1986; 96: 573-5.

8. Kerr-Pontes LRS, Rouquayrol MZ. Medida de saúde coletiva. In: Rouquayrol MZ, organizador. Epidemiologia \& saúde. Rio de Janeiro: MEDSI; 2003. p. 61-82.

9. Cartlidge PHT, Stewart JH. Effect of changing the stillbirth definition on evaluation of perinatal mortality rates. Lancet. 1995; 346: 486-8.

10. De Lorenzi DRS, Tanaka ACD, Bozzetti MCB, Ribas FE, Weissheimer L. A natimortalidade como indicador de saúde perinatal. Cad Saúde Pública. 2001; 17: 141-6.

11. OMS (Organização Mundial da Saúde). Classificação Estatística Internacional de Doenças e Problemas Relacionados à Saúde. 10. rev. São Paulo: EDUSP; 1993.

Recebido em 1 de outubro de 2007

Versão final apresentada em 25 de março de 2008

Aprovado em 16 de junho de 2008
12. Laurenti R, Buchalla CM. Indicadores de saúde materna e infantil: implicações da 10D. rev. da Classificação de Doenças. Rev Panam Salud Pública. 1997; 1: 18-22.

13. Laurenti R, Buchala CM, Lolio CA, Santo AH, Jorge MH Mortalidade de mulheres em idade fértil no Município de São Paulo. Rev Saúde Pública. 1990; 24: 128-33.

14. Mello Jorge MHP, Gotlieb SLD, Soboll MLMS, Almeida MF, Latorre MRDO. Evaluation of the information system on live-births and of the use of its data in epidemiology and health statistics. Rev Saúde Pública. 1993; 27 (Suppl); 146.

15. Litle RE, Weinberg CR. Risk factors for antepartum and intrapartum stillbirth. Am J Epidemiol. 1993; 137: 1177-89.

16. OMS (Organização Mundial da Saúde). Centro Colaborador para Doenças em Português. Universidade de São Paulo. Classificação estatística internacional de doenças e problemas relacionados à saúde. 9. rev. São Paulo; 1980

17. Szwarcwald CL, Leal MC, Castilho EA. Mortalidade infantil no Brasil: Belíndia ou Bulgária? Cad Saúde Pública. 1997; 13: 503-16

18. Laurenti R, Jorge MHP, Lebrão ML, Gotlieb SLD, Almeida MF. Estatísticas vitais: contando nascimentos e mortes. Rev Bras Epidemiol. 2005; 8: 108-10.

19. Siqueira AAF, Rio EMB, Tanaka ACD, Schor N, Alvarenga AT, Almeida LC. Mortalidade feminina na região sul do município e São Paulo: qualidade da certificação médica dos óbitos. Rev Saúde Pública. 1999; 33: 499-504.

20. Ximenes FMA, Oliveira MCR. A influência da idade materna sobre as condições perinatais. Rev Bras Prom Saúde. 2004; 17: 56-60.

21. Gama SGN, Szwarcwald CL, Leal MC, Theme Filha MM. Gravidez na adolescência como fator de risco para baixo peso ao nascer no município do Rio de Janeiro, 1996 a 1998. Rev Saúde Pública. 2001; 35: 74-80.

22. Nascimento LFC, Gotlieg SLD. Fatores de risco para o baixo peso ao nascer, com base em Informações da Declaração de Nascido Vivo em Guaratinguetá, São Paulo, no ano de 1998. Inf Epidemiol SUS. 2001; 10: 113-20.

23. Gripp GBM, Barros Filho AA. A gravidez na adolescência é fator de risco para o baixo peso ao nascer? (Brasil). Rev Chil Pediatr. 2000; 71: 453-60.

24. Almeida MF, Mello Jorge MHP. Pequenos para idade gestacional: fator de risco para mortalidade neonatal. Rev Saúde Pública. 1998; 32: 217-24. 